\title{
A behavioral frequency discrimination paradigm for use in adult primates
}

\author{
GREGG H. RECANZONE, WILLIAM M. JENKINS, GARY T. HRADEK, \\ and MICHAEL M. MERZENICH \\ University of California, San Francisco, California
}

\begin{abstract}
A psychophysical apparatus and procedure that can be used to define the performance and estimate the thresholds of frequency discrimination in adult primates is described. This protocol has been used to train adult owl monkeys to discriminate differences in the frequencies of successively presented sinusoidal tactile stimuli delivered to a restricted skin surface of a single digit, and to train a second set of adult owl monkeys to discriminate differences in frequencies between paired auditory tonal stimuli. The thresholds estimated with this procedure are comparable to those measured in other primates. The advantages of using this procedure for combined behavioral and physiological studies are discussed.
\end{abstract}

Adult owl monkeys (genus Aotus) are a valuable model for electrophysiological and anatomical studies of the neocortex. These primates have been used in studies of the basic organization of auditory, somatosensory, and visual cortical areas (Allman \& Kaas, 1975, 1976; Imig, Ruggero, Kitzes, Javel, \& Brugge, 1977; Kaas, Nelson, Sur, ChiaSheng, \& Merzenich, 1979; Merzenich \& Kaas, 1980; Merzenich, Kaas, Sur, \& Lin, 1978; Merzenich et al., 1987). The functional organization of the cerebral cortex in the owl monkey has been studied in detail, yet only limited psychophysical data are available for this species. Cortical plasticity studies of the owl monkey and other species have demonstrated that the cortical representation of the body surface in somatosensory cortical area $3 \mathrm{~b}$ can undergo extensive reorganization following a variety of surgical manipulations (see Merzenich, Recanzone, Jenkins, Allard, \& Nudo, 1988; Merzenich, Recanzone, Jenkins, \& Nudo, 1990) or by mechanical stimulation of a restricted skin surface (Jenkins, Merzenich, Ochs, Allard, \& Guice-Robles, 1990). Similar observations have been made of auditory (Robertson \& Irvine, 1989), visual (Kaas et al., 1990), motor (Sanes, Suner, \& Donoghue, 1990), and second somatosensory cortex (SII; Pons, Garraghty, \& Mishkin, 1988) in other species. These studies have

The authors would like to thank Lazlo Bocskai, Burton Rutkin, and Marshall Fong for technical support in development of the apparatus. We would also like to thank Henry Heffner for insightful discussion of the temporal parameters for the stimulus presentation, advice in developing the probability tables of $\$ 2$ presentation, and assistance in the safe-rate correction procedure. We are grateful to $K$. $O$. Johnson for the generous use of a Chubbuck tactile stimulator, and Thomas Chimento for critical comments on earlier versions of the manuscript. This project was funded by NIH Grants NS-10414 and GM-07449, and by Hearing Research and the Coleman Fund. G. H. Recanzone is presently at the Laboratory of Sensorimotor Research, National Eye Institute, National Institutes of Health, Bethesda, MD 20892. Correspondence should be addressed to Michael M. Merzenich, HSE 871 Box 0732, University of California, San Francisco, CA 94143-0732. led to the hypothesis that cortical representational topographies are dynamic, and that they are shaped in their details by behavioral experience. One prediction of this hypothesis is that the central representation of a sensory surface would be altered following a period of training of that sensory surface. This change in the central representation of the sensory surface may occur in parallel with the increase in performance commonly observed with training (see Anderson, 1981; Gibson, 1953; James, 1890/1950; Singley \& Anderson, 1989).

Any psychophysical procedure with which this prediction could be tested would have to meet at least four requirements: (1) It must permit the generation of a learning curve. (2) It must permit the study of trained and untrained sensory receptor epithelia in the same individual, so that improvements in performance due to learning an effective strategy, which should generalize across sensory epithelia, can be differentiated from improvements attributable to the hypothesized central reorganizations resulting in increased acuity, which should be more specific to the trained sensory epithelia. (3) The trained receptor surface should receive extensive stimulation during the course of training and testing, because the central reorganizations are presumed to be driven by use (i.e., stimulation). (4) It must be possible to modify the procedure easily for use with other species, particularly humans, in order to make direct comparisons between them.

One psychophysical procedure that meets each of these requirements is a modified go/no-go procedure that has allowed us to define frequency discrimination thresholds in the somatosensory and auditory systems of adult owl monkeys and humans. This procedure has many advantages, including the following: (1) The subject receives a large number of stimulus presentations. (2) Stimulation can be restricted to precisely the same skin region or auditory frequency in each session. (3) The performance at several different frequencies can be estimated daily for a variable training period. (4) Training can be accom- 
plished in 3-5 months. (5) Transference to alternative frequencies and/or skin surfaces in the same individual is straightforward. (6) The degree of attention required for successful completion of the task can be varied by changing either the amplitude of the stimuli or the range of differences in the frequency of the $S 2$ stimuli. (7) Other aspects of sensory discrimination, such as amplitude or duration, can easily be adapted to this procedure. (8) Finally, this paradigm is suitable for use on any primate with only minor modifications, and it is easily adapted for use in humans.

We have used this paradigm to train 9 individual adult owl monkeys in a tactile or auditory frequency discrimination paradigm. The results of these behavioral and physiological studies are the subject of a series of reports described elsewhere (Recanzone, Jenkins, Hradek, \& Merzenich, 1991; Recanzone, Merzenich, \& Jenkins, 1991; Recanzone, Merzenich, Jenkins, Grajski, \& Dinse, 1991; Recanzone, Merzenich, \& Schreiner, 1991). Here, we will focus on the behavioral methods developed for these experiments.

\section{METHOD}

\section{Animals}

Feral owl monkeys of both sexes were used for these experiments. The animals were judged to be adult by their body weight $(900-1,500 \mathrm{~g})$ and overall appearance. The animals were housed in individual cages on a reverse 12:12-h light:dark cycle (lights on at 7:00 p.m.). Ad-lib body weight was determined at the start of the training period. The monkeys were maintained at $85 \%-95 \%$ ad-lib body weight throughout the course of training and testing.

\section{Apparatus}

Internal apparatus. The internal apparatus was housed inside a single-walled acoustic chamber (Lehigh Valley Electronics; see Figure 1A). The inside walls and door of the chamber were covered with 4-in.-thick echoattenuating foam. The cage was constructed of 3-mmdiameter stainless steel rods separated by $27 \mathrm{~mm}$; the overall dimensions were $28 \times 21 \times 48 \mathrm{~cm}$. The cage was fastened to the floor of the chamber on a $2-\mathrm{cm}$ foam pad to reduce vibrations. The animal extended its hand and arm outside the cage through a $4.5 \times 14 \mathrm{~cm}$ opening to make contact with the hand mold.

The hand molds were constructed from polyform sheet. Each mold was shaped with a shallow groove to accommodate each digit. The palm-digit angle was approximately $105^{\circ}$ when the digits and palm rested on the mold (see Figure 1B). Strips of gold-plated copper foil $(3 \times 10 \mathrm{~mm})$ were imbedded in each groove with cyanoacrylate to underlie the distal phalanx of each digit, with one placed under the base of the palm.

Openings were made in the mold to allow access to the skin surfaces of interest. The stimulator probe was a 2mm-diameter monofilament rod with a hemispherical tip, which contacted the skin at a $90^{\circ}$ angle through the opening. The tactile stimulator delivered sinusoidal waveforms according to descriptions provided by Chubbuck (1966).

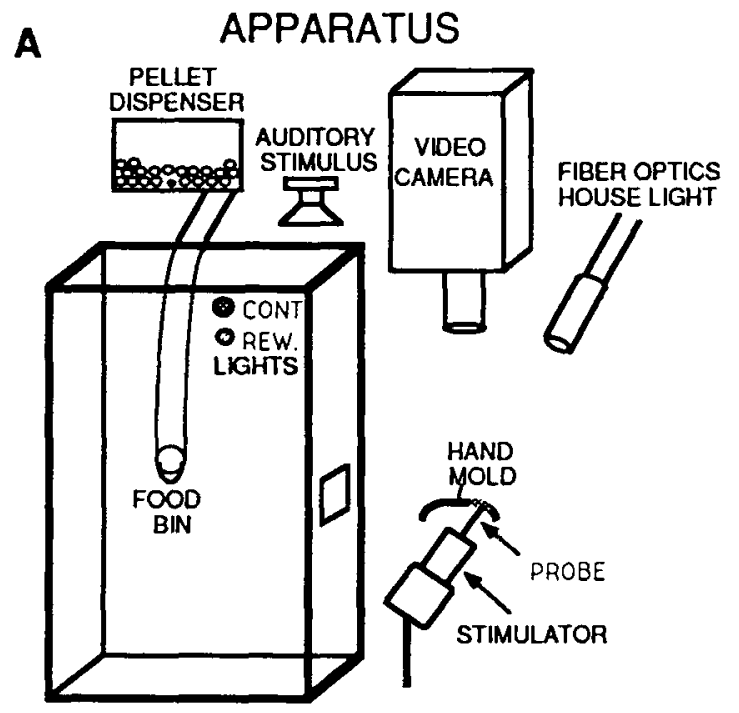

B HAND MOLD

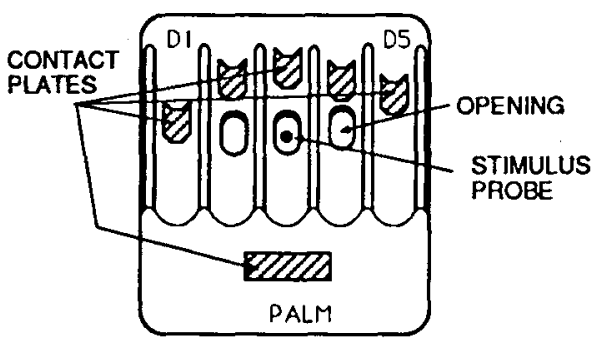

Figure 1. Schematic diagram of the psychophysical apparatus (A) and the hand mold (B). The animal was trained to make contact with the hand mold by reaching through the small opening in the front of the test cage. The bars of the test cage have been omitted from this diagram for clarity. The tactile stimulator contacted the animals skin through an opening in the hand mold. The video camera provided the investigator with on-line visual monitoring of the monkey's hand position. The auditory stimulus was placed directly overhead, above the animal. The hand mold was constructed so that the animal's hand could be in only one position in order to activate all of the six contact plates. The stimulus probe was usually positioned to contact the middle phalanx on the third digit. (See text for details.)

Rewards were 45-mg banana-flavored food pellets with vitamin and mineral supplements (Bio-Serve, FO382). The number of pellets eaten in a normal session (approximately 400) contained an equivalent amount of all major vitamins, minerals, and salts when compared to the normal diet of freely available chow biscuits (Purina). The reduced caloric count to maintain body weight at $85 \%-95 \%$ was represented almost entirely by a reduction in the fat content of the pellets. The automated pellet dispenser (Gerbands D-1) was located outside of the test chamber. A fiberoptic light source directed onto the hand mold was the main source of illumination within the acoustic chamber and served as the houselight. Two $6.5-\mathrm{V}$ incandescent lights of different colors were located at the top of the 
cage. A video camera was used for continual visual monitoring of the monkey's hand position.

External apparatus. An Apple II microcomputer with digital interfaces (Jenkins, 1982) controlled the hardware associated with the chamber. The computer software controlled all aspects of the behavioral procedure, provided on-line feedback regarding the animals' performance, and allowed the experimenter to adjust several stimulus parameters without interruption. The stimuli consisted of sinusoidal waveforms generated with custom waveform generation electronics. The auditory stimulus was amplified (Crown D76) and presented through a conventional speaker (Realistic 40-1996). The amplifier output and displacement feedback from the tactile stimulator were monitored on an oscilloscope (Hameg). At the start of each session, and before the subject was placed in the test chamber, a series of standard frequency/amplitude waveforms for either tactile or auditory stimuli were presented and their voltage outputs were matched to all previous sessions. The intensity of auditory stimuli at the frequencies presented were measured at 12-24 locations within the test chamber cage (Brüel \& Kjær sound-level meter, Type 1613). These locations evenly sampled the threedimensional space occupied by the monkey's head during a normal session. These measurements were made at benchmark times throughout the several weeks of training and testing, and they did not reveal any significant

The contact circuitry delivered a step voltage upon detection of $\leq 5 \mathrm{M} \Omega$ of resistance between the contact plates and the grounded cage floor. The experimenter could set any combination of inputs corresponding to the individual digits or palm as "correct" to initiate a stimulus run. This combination of inputs was selected in such a way that the animal could make correct contact by only one hand position.

Prior to the start of each trial, the computer determined the frequency and the bin in which the $\$ 2$ stimulus would be presented. Once correct hand position was established, the stimuli were presented while the computer monitored the hand position. During the course of the session, the computer displayed the number of presentations and the hit rate for each S2 stimulus, the false-positive rate for each stimulus bin, the overall false-positive rate, and the reward rate, defined as the number of rewards divided by the number of trials. The experimenter could change the values of the miss and false-positive time-outs, the range of S2 frequencies presented, and most other stimulus parameters. In practice, the session was initiated with all presented $\mathbf{S} 2$ frequencies well above threshold. This warm-up period lasted 5-10 min. The range of S2 frequencies presented could then be adjusted to the range used to generate the data on which estimates of threshold were based. Once this final range was set, the experimenter was not required to interact with the session until completion.

\section{Procedure}

The psychophysical procedure was similar for the somatosensory and auditory paradigms. The somatosen- sory task will be described first, followed by the minor alterations used in the auditory task. The techniques used to train naive monkeys to perform these tasks will be described in a later section.

Tactile frequency discrimination. The tactile frequency discrimination protocol is diagrammed in Figure $2 \mathrm{~A}$. The subject initiated each run by making correct contact with the hand mold, signaled by the contact circuit output. The subject received feedback that the hand was placed correctly from the illumination of an indicator light. A 1-sec prestimulation period began in which the animal could break correct contact without penalty. This preperiod was followed by a ramped displacement of the stimulator probe onto the skin surface. All sinusoidal stimulation for the run was superimposed onto this ramped displacement. A completed run consisted of two to six stimulus presentations (bins) of 650 -msec duration with a $650-\mathrm{msec}$ pause between bins. In each stimulus bin, the probe was set into sinusoidal oscillation at a frequency of $20-40 \mathrm{~Hz}$. The first stimulus presented was always the comparison stimulus (S1). Presentations 2-5 had an equal probability ( 0.388$)$ that the stimulus would be a higher frequency ( $\mathbf{2} 2$, given that the $\mathbf{S} 2$ had not yet been presented. Thus, the probability that an $\mathbf{S 2}$ would occur in the next bin did not increase during a run (see below). Rewards were obtained only on runs in which correct hand contact was maintained through the $S 1$ stimulus presentation(s) and broken within an 800-msec reward window. The reward window began $150 \mathrm{msec}$ after $\mathrm{S} 2$ onset and ended $300 \mathrm{msec}$ after $\mathrm{S} 2$ offset $(800 \mathrm{msec})$. The fastest reaction time recorded for this task in these animals was more than $150 \mathrm{msec}$; thus the animal could not receive a reward for "guessing" at the start of a stimulus run. The reward window ended prior to the expected onset of the next stimulus; thus the "missed onset" of the next stimulus could not be used as a cue. Rewards were delivered simultaneously with the illumination of a second indicator light. All lights were extinguished for a set period (miss time-out) and no reward was delivered if the subject did not break contact during the 800-msec reward window. Releases made prior to the hit window resulted in a false-positive time-out.

An important requirement of this paradigm is that correct hand placement be maintained throughout the stimulus presentation. The relatively small contact detector surfaces, and the requirement that many of them be simultaneously activated, meant that even very small movements were detected as a release. All well-trained monkeys developed a stereotyped and very stable hand position for the hand placement behavior, as well as a very stereotypical response, which invariably entailed the rapid and complete withdrawal of the hand from the hand mold.

In order for the probability that an S2 stimulus would occur in the next stimulus bin to remain constant within an individual trial, the bin in which the $\mathbf{S} 2$ stimulus was presented was selected from one of eight lookup tables. These lookup tables were created by using the values in Table 1 in pseudorandom order, with the provision that 
A Tactile Frequency Discrimination Paradigm

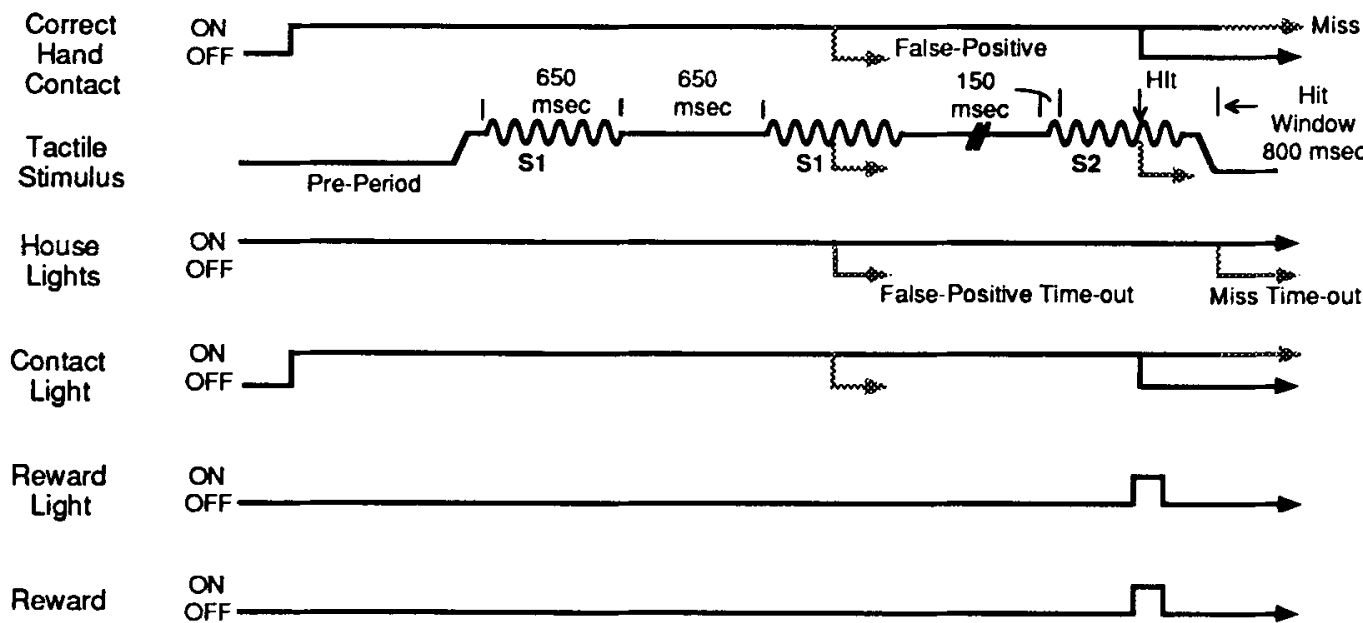

$\mathbf{B}$ Auditory Frequency Discrimination Paradigm

Correct Hand

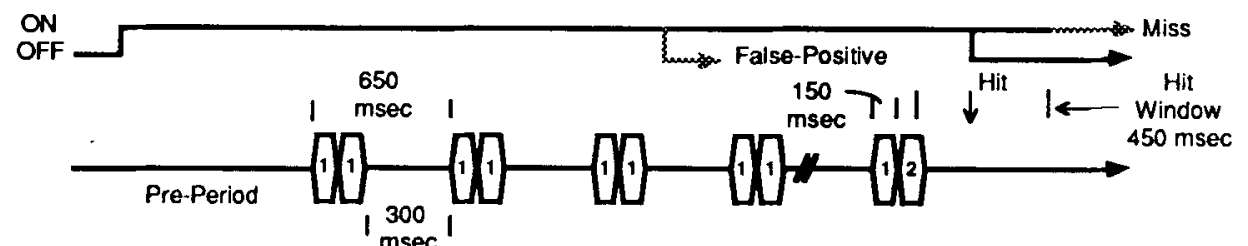

House

Lights

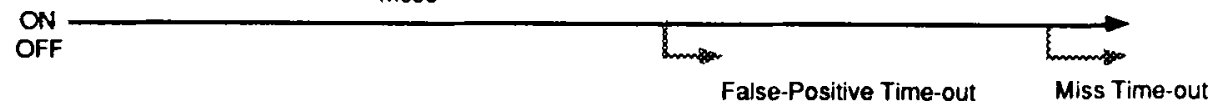

Contact
Light

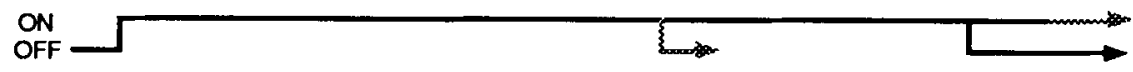

Reward
Light

ON

OFF

Reward

ON

OFF

Figure 2. The psychophysical paradigm for the tactile task (A) and the auditory task (B). In both tasks, each run was initiated by the subject's making correct hand placement. For the tactile task, the stimuli were presented as bursts of sinusoidal stimulation for $650 \mathrm{msec}$, with a 650 -msec pause between stimuli. The first stimulus was always the comparison frequency (S1). The S2 was higher in frequency. False-positive time-outs resulted when the animal released the hand mold before the presentation of the $S 2$ stimulus. Miss time-outs resulted when the animal did not release during the 800 -msec reward window. The auditory task was similar, except that the stimuli consisted of a pair of tones, 150 msec in duration and separated by 50 msec. These tone pairs were separated by 300 msec. The S1 consisted of both tones at the same frequency; for the S2, the second tone pip of the pair was higher in frequency. 
Table 1

Probability of S2 Occurrence by Bin

\begin{tabular}{ccc}
\hline $\begin{array}{c}\text { Bin } \\
\text { of Presentation }\end{array}$ & $\begin{array}{c}\text { No. } \\
\text { Occurrences/100 }\end{array}$ & Probability \\
\hline \multicolumn{3}{c}{ Tactile Stimulus } \\
2 & 37 & 0.37 \\
3 & 25 & 0.40 \\
4 & 15 & 0.39 \\
5 & 9 & 0.39 \\
6 & 14 & 1.00 \\
$M \pm S D$, Bins 2-5 & & $0.388 \pm 0.012$ \\
& Auditory Stimulus & \\
2 & 0 & 0.00 \\
3 & 31 & 0.31 \\
4 & 22 & 0.32 \\
5 & 15 & 0.32 \\
6 & 10 & 0.31 \\
7 & 7 & 0.32 \\
8 & 5 & 0.33 \\
9 & 3 & 0.30 \\
10 & 2 & 0.29 \\
11 & 3 & 0.60 \\
12 & 2 & 1.00 \\
$M \pm S D$, Bins 3-10 & & $0.312 \pm 0.014$ \\
\hline
\end{tabular}

Note-Values are for the eight lookup tables that were used to determine the bin of presentation of the $\mathbf{S} 2$ stimulus. The probability was calculated as the probability that the next stimulus would be the S2 stimulus given that the $\mathbf{2} 2$ had not yet occurred. (See text for details.)

the same bin of $\mathbf{S} 2$ presentation was not repeated on more than three consecutive runs. Four of these tables were randomly selected to run in sequential order for each session, but the same table was never used as the first table on 2 consecutive days. The $\$ 2$ presentation bin was selected in sequential order from the lookup tables. If the animal made a false-positive response, the same $\mathbf{S} 2$ was repeated for the next run, but in the bin of presentation next in order from the lookup table. Thus the $\mathbf{S 2}$ was presented in a different sequence of bins, depending on the false positives for a given session. This feature, in combination with the variable set of possible combinations of tables, prevented the animal from responding to the $\$ 2$ presentations on the basis of learning the lookup table sequences.

Catch trials. Two types of catch trials were used in each session to ensure that the animal was discriminating the change in stimulus frequency. In order to keep the probability of $\mathbf{S} 2$ occurrence constant across bins, it was necessary to make the sixth bin always present the $S 2$ (see Table 1). These runs were not counted in the data analysis, since they could be predicted on the basis of elapsed time. We also imposed a $.005-.01$ probability that the S2 stimulus would be equal to the $S 1$ stimulus. In these runs, an animal under stimulus control did not respond. These few catch runs each day were important indicators of "guessing"' or "timing" strategies (see below) and were used to empirically define chance performance. In a different class of catch trials, we also moved the stimulus probe off the skin on a few runs (3-5) in approximately 1 out of 10 sessions, to ensure that the animal was not using other cues to discriminate the stimulus. The animals under stimulus control did not respond in this condition. In all three types of catch trials, if the monkey did make a response during the presentation of the $\mathbf{S} 2$ stimulus, it was rewarded with a banana pellet. In practice, for either the catch trials in which the $S 2$ stimulus was equal to the $S 1$ stimulus or in which the stimulus probe was moved off the skin, the animals did not release the hand mold on these few trials per session and thus were not rewarded.

Daily session. Each session was initiated with a warmup period consisting of 2-4 S2 frequencies that were above $90 \%$ correct in the previous session. The animal was allowed to obtain 50-100 rewards at these S2 frequencies. This procedure was necessary, because most of the animals had a tendency to make false-positive responses if they were not rewarded at a high rate at the start of a session. The set of $\mathbf{S} 2$ frequencies was then gradually changed over the next 50-100 rewards, ultimately to a frequency range of 6-10 frequencies, with approximately 2 below the $50 \%$ correct rate. The order of $\mathbf{2} 2$ frequency presentation was randomized for each session. We targeted the tested frequencies to ensure an overall reward rate (no. of rewards/no. of runs) at approximately $60 \%$ or greater, with an overall false-positive rate of $15 \%$ or less. A daily session consisted of the number of runs required to deliver approximately 400 rewards.

Auditory frequency discrimination. The auditory discrimination paradigm is diagramed in Figure 2B. The auditory stimuli were presented as pairs of tones. Each tone pip was $150 \mathrm{msec}$ in duration with a 10 -msec on-off ramp. Each tone pip of the pair was separated by $50 \mathrm{msec}$. These pairs were presented every $650 \mathrm{msec}$, with a maximum of 12 stimulus presentation bins. In the auditory paradigm, the first two pairs of tone pips were the S1 stimulus. For Bins 3-11, the probability of the next bin's being the $\mathbf{S 2}$ stimulus, provided that the previous bin was an S1, was approximately 0.312 (see Table 1). The two tone pips were identical in frequency for the $\$ 1$ stimulus. For the S2 stimulus, the second tone pip in the pair was higher in frequency. The amplitude of the first tone pip relative to the second varied randomly over $12 \mathrm{~dB}$ in 3-dB steps for each pair of tone pips. The response window in this paradigm began $100 \mathrm{msec}$ after tone onset and ended $400 \mathrm{msec}$ after tone offset (total, $450 \mathrm{msec}$ ).

Shaping. A three-stage strategy was used to train the owl monkeys. Initial screening of owl monkeys was performed by providing 100 pellets each day in addition to the usual diet of chow biscuits and ad-lib fluid enriched with vitamin $C$ (Tang, General Foods). If the animal showed little or no interest in the food pellets, they were eliminated from the study. All hardware used at each stage of training was made as uniform as possible to aid in the transition between stages. The first stage was designed to train the animals to make the appropriate contact with the hand mold, so that the stimulus presentation could be restricted to an invariant location. The second stage was designed to train the animal in a stimulus-detection task, and the final stage was designed to train the animal in the discrimination task. The animals were trained for about 
$2 \mathrm{~h}$ a day for 6 days a week. The training procedure was virtually identical for both the auditory and the tactile frequency discrimination paradigms, with the exception of the stimulus modality. The following describes the procedure used in training monkeys at the tactile discrimination task. The minor differences for the auditory task are described later.

Tactile discrimination training. In the first stage, the animals were shaped to contact a hand mold. Ad-lib body weight was determined for the selected subjects. The Tang was replaced with freely available water. Chow biscuits and fruit were provided as supplements to the food-pellet diet only as was necessary, to maintain body weight between $85 \%-110 \%$ ad lib.

A hand mold and pellet dispenser were attached to the outside of the home cage in the animal colony. The apparatus was available to the animal throughout the day, and care was taken to ensure that the animal received sufficient nutrition. Initially, contact with any part of this hand mold illuminated an indicator light and delivered a food pellet. The animal was then shaped to make contact with each of three $3 \times 5 \mathrm{~mm}$ copper foil strips placed to underlie Digits 2, 3, and 4 when the hand was placed squarely on the mold. When the animal routinely received 300-400 pellets in less than $2 \mathrm{~h}$, commonly within 3 weeks, a limited hold requirement was added. The indicator light provided feedback that the hand was in the correct position. This position had to be maintained for the limited hold period before the pellet dispenser was activated. Breaking contact early resulted in resetting the limited hold time. The limited hold period started at $100 \mathrm{msec}$ and was slowly increased over a period of several weeks, ultimately to $5 \mathrm{sec}$. This initial stage of training was done in the animals' home cages, to familiarize the animal with the food hopper and manipulanda. This familiarization facilitated the transfer to the training apparatus used in the second stage.

For the remainder of the training, the animal was transported via a small opaque transport box to a training apparatus housed inside a single-walled acoustic chamber. The training apparatus was a simplified version of the testing apparatus described in detail above. It was necessary to transfer the monkey to this second apparatus in order to deliver the tactile or auditory stimuli, and to reduce the distractions in the monkey's environment. The hand mold in this apparatus had six $2 \times 4 \mathrm{~mm}$ copper foil strips located to underlie each of the five digits and the center of the palm when the hand was centrally placed. The animals were allowed several sessions to become acclimatized to the new environment and hand mold. They quickly adopted a stereotypical hand position. The digit and skin site to be stimulated were then selected, and an opening was made in the hand mold to allow the tactile stimulator to contact the selected skin surface.

In the second stage, the animals were trained to perform a variable hold detection task. In the initial procedure, a $40-\mathrm{Hz}, 2-\mathrm{mm}$ peak-to-peak stimulus was presented following a variable hold period of $1-3 \mathrm{sec}$. This stimu- lus remained on until the animal released the hand mold. The duration of the stimulus presentation was recorded for each run, and the average time was displayed. Once the mean response time fell below $2 \mathrm{sec}$, which commonly occurred within three to five sessions, the variable hold period was gradually increased to 3-5 sec.

In the final stage, the animals were trained to perform a frequency discrimination. Following correct hand placement, a short fixed delay followed in which contact could be broken without penalty. A 5-Hz, 1-mm peak-to-peak stimulus was then presented for $650 \mathrm{msec}$, followed by a 650-msec pause. The animal was not rewarded if contact was broken during the presentation of this stimulus (S1). The S1 was initially presented one or two times before the $40-\mathrm{Hz}$ stimulus (S2) was presented, and it remained on until contact was broken. Once the animal could easily make this discrimination, the $\$ 2$ presentation time was gradually decreased to $650 \mathrm{msec}$. The reward window (see above) was set to $800 \mathrm{msec}$. A miss time-out was imposed by turning off the houselight for $2 \mathrm{sec}$ following a miss. The number of S1 stimuli presented before the $\$ 2$ was gradually increased to five. A false-positive time-out was also imposed, in which the houselight was turned off for $2 \mathrm{sec}$ following a response during the $S 1$ presentation.

Once the animal was consistently making these easy discriminations, the amplitude of the $\mathrm{S} 2$ stimulus was decreased to match that of the $S 1$. The $S 1$ frequency was then increased to $20 \mathrm{~Hz}$ over the next several sessions. The animal was allowed several sessions $(3-10)$ in which it consistently made the $20-\mathrm{Hz}$ versus $40-\mathrm{Hz}$ discrimination. Finally, the stimulus amplitude was reduced to $300 \mu \mathrm{m}$ peak to peak. At this point the animal was considered trained, and it was moved to the testing apparatus. In our experience, a single investigator can run only 2-3 monkeys in a single apparatus each day. By using a testing and training apparatus, as well as the on-cage device used in the first stage of training, several animals at different stages of training as well as 2-3 fully trained animals could be run by a single investigator on a given day. However, all of the training and testing could have been accomplished in only the testing apparatus.

Auditory discrimination training. The auditory discrimination training was relatively less difficult, in that it was not critical that the hand be placed in an identical manner on each trial. Thus the hand mold could be replaced with a simple mechanical lever, and correct hand placement could then be defined as a leverpress.

The initial parameters for the detection task were based on the target S1. The S2 stimulus was initially set at 2-3 $\mathrm{kHz}$ greater than the target $\mathrm{S} 1$ stimulus. The animal was initially required to detect the S2 tone at approximately $70 \mathrm{~dB}$ SPL. Stage 3 shaping was started with an S1 consisting of a 20-dB SPL tone at $1-2 \mathrm{kHz}$ less in frequency than the target $S 1$ mixed with white noise to a combined amplitude of $40 \mathrm{~dB}$ SPL. The white noise was used in auditory stimulation to create a very large and salient difference between the S1 and S2 stimuli (i.e., amplitude, 
frequency, and spectrum). The combined amplitude was first increased to match the $S 2$; then the amplitude of the white noise was reduced, while that of the tone was increased over the course of many sessions until the animal was discriminating between the two tones at equal amplitudes, as in the tactile task. The frequency of the S1 was then increased to the target value, and the $S 2$ was decreased as in the tactile task. Once the animal could reliably discriminate the two different frequencies, it was moved to the testing apparatus.

\section{Data Analysis}

The data stored for each run of a session were: (1) the frequency of the presented S2 stimulus; (2) the duration of the presented $\mathbf{S 2}$ stimulus, measured from the onset of the S2 stimulus to either the response (e.g., a hit) or the termination of the stimulus (e.g., a miss); (3) the duration of the $S 1$ stimulus presentations, similarly measured from the stimulus onset to either the response or the offset for each S1 pulse; and (4) the response (see below). The hit rates and reaction times for each S2 frequency presented, the number and duration of all stimuli presented in that session, and the false-positive rate could be calculated from these values. These calculations could be made over the entire session, within any subset of the session, and with respect to the S2 presentation bin.

Each trial was classified into one of five categories for data analysis:

Hit. Correct contact with the hand mold was broken during the reward window for S2 presentations in Bins $2-5$. Each $S 2$ frequency was counted independently.

Miss. Correct contact with the hand mold was maintained during the presentations of $S 1$ and the reward window for S2 presentations in Bins 2-5. Each S2 frequency was counted independently.

False-positive. Correct contact with the hand mold was broken after the onset of the second S1 presentation but before the reward window.

Pre-false-positive. Correct contact with the hand mold was broken before the onset of the second stimulus presentation, either $\mathrm{S} 1$ or $\mathbf{S 2}$. The stimuli presented in this time were always the S1 stimulus, and thus these runs were not used to compute the false-positive rate. The animal received a short time-out for this response.

Catch. Correct contact with the hand mold was maintained to the last stimulus presentation (Bin 6). The animal was rewarded with a correct response, or punished with a miss time-out as appropriate. These runs were not included in the data analysis, since they always consisted of presentations of the $\mathbf{S 2}$ stimulus.

All the various types of catch trials (see above) were treated independently and were not used in the analysis of performance or the threshold estimates.

\section{Estimation of Threshold}

The estimation of threshold was dependent on two parameters: the hit rate at each presented S2, and the false- positive rate. The hit rate for a given S2 frequency was defined as follows:

$$
\mathrm{H}_{s 2}=\frac{\text { no. correct responses }}{\text { no. stimulus presentations }} .
$$

The data used in this equation were pooled from runs in which the given $\mathbf{2} 2$ frequency was presented in Bins 2-5. The false-positive rate was calculated for each bin $n\left(F_{n}\right)$ as follows:

$$
\mathrm{FP}_{n}=\frac{\text { no. responses made to presentations of } S 1 \text { in bin } n}{\text { no. presentations of } S 1 \text { in bin } n} \text {. }
$$

Through comparison of the false-positive rates for each bin, a "late-guessing" strategy could be identified (see below). The false-positive values for each bin were always comparable when the animal was under stimulus control. The total false-positive rate for the session, $\mathrm{FP}_{t}$, was then derived by

$\mathrm{FP}_{t}=\frac{\text { total no. responses made to presentations of } \mathrm{S} 1}{\text { total no. presentations of } \mathrm{S} 1 \text { in Bins 2-5 }}$

The overall performance rate for a given $\$ 2$ frequency $\left(P_{S 2}\right)$ could then be calculated by using the hit rate for that frequency with correction for the false-positive rate. For simplicity's sake, the correction term was the "safe" rate $(\mathrm{S})$, defined as follows:

$$
S=1-F P,
$$

and thus the performance $(\mathrm{P})$ for any given $\mathrm{S} 2$ frequency was defined as

$$
\mathbf{P}_{\mathbf{S} 2}=\mathrm{S}_{\mathrm{t}}-\left[\left(1-\mathrm{H}_{\mathbf{S 2}}\right) \mathrm{S}_{t}\right],
$$

where $S_{t}$ is the overall safe rate for all presentation bins. This equation then reduces to

$$
\mathrm{P}_{\mathrm{S} 2}=\mathrm{S}_{\mathrm{t}} \times \mathrm{H}_{\mathrm{S2}} \text {. }
$$

A typical performance function demonstrating the effect of the false-positive rate on threshold estimates is shown in Figure 3. Panel A shows data taken from a welltrained owl monkey and shows the raw performance function (solid symbols) and the corrected function (open symbols). This animal had a false-positive rate of $8 \%$. The threshold estimate was only slightly increased when the correction was made for the false-positive rate $(0.14 \mathrm{~Hz}$, or $5.6 \%$ of $2.48 \mathrm{~Hz}$ ). Panel B shows the same raw data with theoretical false-positive rates of $5 \%, 10 \%, 15 \%$, and $20 \%$. False-positive rates above $15 \%$ result in a much higher estimate of threshold $(11.7 \%$ and $16.5 \%$ of $2.48 \mathrm{~Hz}$ for false-positive rates of $15 \%$ and $20 \%$, respectively). In addition, animals performing at a high false-positive rate were commonly not under strict stimulus control, as high false-positive rates were characteristic of the timing strategies described below. We excluded all data in which the overall false-positive rate exceeded $15 \%$.

This measurement of threshold provides a simple, quantified result with which to summarize the performance of 
A

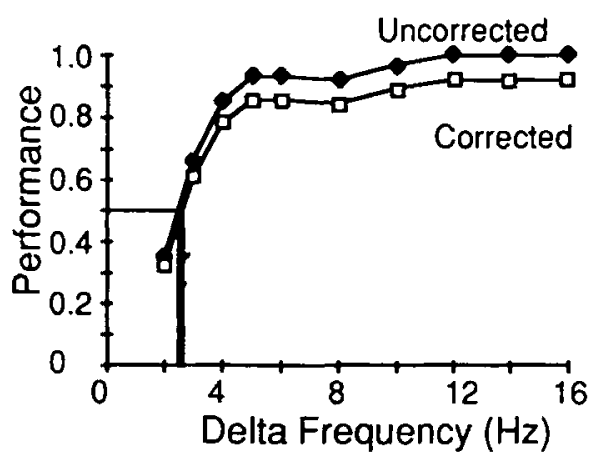

B

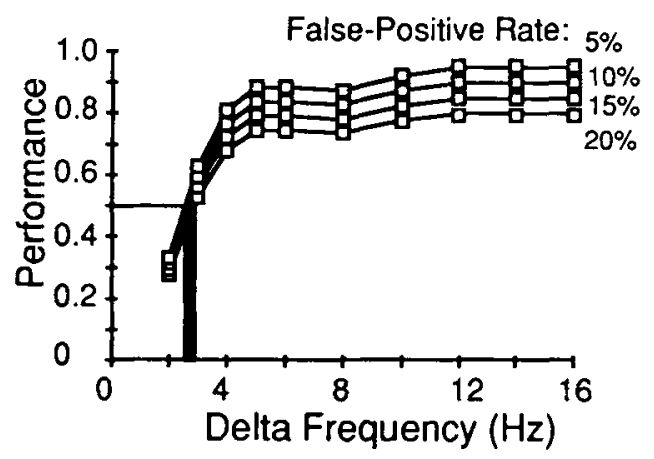

Figure 3. Psychophysical performance functions from a single animal, showing the effects of the false-positive rate on the threshold estimate. In Panel A, the raw performance function is shown by filled symbols (uncorrected). The delta frequency is measured relative to a 20-Hz comparison frequency. From this psychometric function, the threshold was estimated to be $2.48 \mathrm{~Hz}$. The same data corrected by the false-positive rate (see Method) is shown by open symbols (corrected). The estimated threshold from this psychometric function was $2.62 \mathrm{~Hz}$. Panel B shows the same data at four different theoretical false-positive rates. The estimates of threshold were 2.57, $2.66,2.77$, and $2.89 \mathrm{~Hz}$ for false-positive rates of $5 \%, 10 \%, 15 \%$, and $20 \%$, respectively. (See text for details.)

a single session. The correction of each hit rate provides a realistic adjustment to the small variations in the falsepositive rates seen across sessions in an individual monkey. This threshold measure can also be used to compare the results defined by this paradigm with those of other investigators and other species (see below). A main concern, however, is to ensure that this measure is an accurate reflection of the animal's ability to discriminate very small differences in frequency.

A potential problem of the method outlined above is that at very small delta frequencies, which the animal cannot detect as different, the hit rate should be about the same as the false-positive rate. For low false-positive rates, this would lead to an estimated performance for that frequency to be very near the false-positive rate itself, which could in fact be an overestimate of the actual discriminable performance. However, this concern is negligible near threshold, where uncorrected performance values range from approximately $30 \%-70 \%$ with false-positive rates on the order of $8 \%$. In this condition, the performance values are much greater than the false-positive rate (3.5- to 9-fold), and the calculation of threshold is not significantly affected.

An alternative method of analyzing these data is to use signal detection theory and calculate a $d^{\prime}$ value for each S2 frequency presented. This method provides more information than the simple threshold calculation does, because a measure of discriminability for each S2 frequency can be obtained. This is clearly the method of choice if one is interested in a more detailed description of the performance, and it has been used to describe improvements in performance with training at the tactile task (Recanzone, Jenkins, et al., 1991). However, it does require considerably more calculation time in comparison with the simple threshold calculation.

Finally, it is important to be certain that the adjusted threshold measurement is a valid measure in comparison with other methods. We approached this issue by comparing the threshold measured with safe-rate correction and with no correction. In order to generate reasonable estimates of the data derived in a single session, the mean false-positive rate and the mean raw hit rates for the two S2 frequencies bracketing the 0.50 level were taken as standard hit and false-positive rates. These values were calculated on the basis of 20 sessions for each of the 3 monkeys trained in the auditory task and 3 representative monkeys trained in the tactile task (Table 2). Signal detection analysis was then used to define the hit rate at each false-positive rate that had the same value of $d^{\prime}$ as the standard. The performance at each of these hit-rate: false-positive-rate pairs was also defined as described above $\left(\mathrm{P}_{S_{2}}\right)$. An example is shown in the top panel of Figure 4, using the standard false-positive rate of 0.12 and standard hit rates of 0.62 for the above-threshold value (squares) and 0.35 for the below-threshold values (diamonds). This monkey (1320) had the highest false-positive rate and also the most variance across sessions in falsepositive rate of all of the monkeys studied. The open symbols show the curve described with the signal-detection method, whereas the closed symbols show the performance defined with the safe-rate correction procedure. The saferate correction procedure had a slope that was much more shallow than that of the curve defined with detection theory analysis. These two curves were in good agreement at low false-positive rates, although the disparity between the curves increases dramatically with increasing falsepositive rates. A serious concern, then, is how this discrepancy is affecting the estimation of threshold.

To address this issue, the hit or performance rate values generated as in the top panel of Figure 4 were used to define the threshold for each false-positive rate. The theory of signal detection states that two stimuli are equivalent if they have the same value of $d^{\prime}$. Plotting thresholds measured by using hit rates calculated to have the same $d^{\prime}$ value as a function of the false-positive rate should 
Table 2

Means and Standard Deviations from Twenty Sessions in Six Representative Monkeys, and the Maximum Possible Error for Thresholds Calculated Using Detection Theory Analysis

\begin{tabular}{|c|c|c|c|c|c|c|c|c|c|}
\hline \multirow[b]{2}{*}{ Case } & \multirow[b]{2}{*}{ Task } & \multicolumn{2}{|c|}{$\begin{array}{c}\text { False-Positive } \\
\text { Rate }\end{array}$} & \multicolumn{2}{|c|}{$\begin{array}{c}\text { Hit Rate above } \\
\text { Threshold } \\
\end{array}$} & \multicolumn{2}{|c|}{$\begin{array}{c}\text { Hit Rate below } \\
\text { Threshold }\end{array}$} & \multirow{2}{*}{$\begin{array}{l}\text { Safe-Rate } \\
\text { Corrected } \\
\text { Maximum } \\
\text { Error }(\mathrm{Hz})\end{array}$} & \multirow{2}{*}{$\begin{array}{l}\text { Uncorrected } \\
\text { Threshold } \\
\text { Maximum } \\
\text { Error }(\mathrm{Hz})\end{array}$} \\
\hline & & $M$ & $S D$ & $M$ & $S D$ & $M$ & $S D$ & & \\
\hline 1677 & Auditory, $2.5 \mathrm{kHz}$ & 0.06 & 0.03 & 0.60 & 0.07 & 0.33 & 0.11 & 5.35 & 11.0 \\
\hline 1705 & Auditory, $2.5 \mathrm{kHz}$ & 0.08 & 0.04 & 0.58 & 0.10 & 0.37 & 0.08 & 6.1 & 14.7 \\
\hline 1328 & Auditory, $8 \mathrm{kHz}$ & 0.06 & 0.04 & 0.69 & 0.09 & 0.27 & 0.14 & 12.9 & 27.0 \\
\hline 1320 & Tactile, $20 \mathrm{~Hz}$ & 0.12 & 0.05 & 0.62 & 0.09 & 0.35 & 0.07 & 0.54 & 1.74 \\
\hline 1321 & Tactile, $20 \mathrm{~Hz}$ & 0.05 & 0.02 & 0.60 & 0.07 & 0.35 & 0.10 & 0.60 & 1.69 \\
\hline 1378 & Tactile, $20 \mathrm{~Hz}$ & 0.07 & 0.03 & 0.59 & 0.06 & 0.34 & 0.12 & 0.88 & 1.66 \\
\hline
\end{tabular}

Note-The faise-positive rate was taken as the overall rate for the entire session. The hit rate above threshold represents the uncorrected hit rate for the first $\mathbf{S 2}$ frequency that was greater than 0.50 . The hit rate below threshold was similarly taken for the first S2 frequency with a hit rate less than 0.50 . These mean values were used as standard values from which the hit rates for a range of false-positive rates were calculated to have the same value of $d^{\prime}$ as the standard (see text and Figure 4). These predicted hit rates were then used to calculate threshold using either the safe-rate correction procedure (column 6) or no correction procedure (column 7). These thresholds are expressed in hertz. The $\$ 1$ frequency is given for each case in column 2.

result in a straight line of zero slope. The thresholds measured with the safe-rate correction method (squares) or no correction method (diamonds) are shown in the bottom panel of Figure 4 for Case 1320. In this monkey, the false-positive rates ranged primarily from 0.07 to a maximum of 0.15 (approximately $\pm 1 S D$ ). This range is shown as the horizontal line. The thresholds measured over this range are fairly uniform, although there is a sharp increase in the slope of the line at very low false-positive rates. By contrast, the thresholds calculated from the same data without the correction factor show a steeper slope over the range of interest and consistently underestimate the threshold at each false-positive rate.

To generate the graphs in the lower panel of Figure 4, the S2 frequency for the above-threshold hit rate was arbitrarily assigned a value of 2.0 , while the S2 frequency for the below-threshold hit rate was assigned a value of 1.0. The potential error of the threshold estimate is therefore not only a function of the false-positive rate but also the step size in frequency of the S2 stimuli. The last two columns in Table 2 show the potential error, in hertz, for each of the 6 representative monkeys. This error was taken as the threshold defined at the false-positive rate of 0.02 subtracted from the threshold at the false-positive rate of 0.15 , and converted to hertz on the basis of the difference in $\mathbf{2} 2$ frequencies actually used in the task. For the safe-rate correction procedure, these maximum errors are limited to only a few hertz in the auditory task and represent approximately $0.2 \%$ of the S1 frequency. In contrast, the uncorrected measure represents an error over twice as large as that with the correction method and represents approximately $0.4 \%$ of the comparison frequency. These errors are relatively small; the $\mathbf{S} 2$ frequencies varied in steps of approximately $7 \mathrm{~Hz}$ for the $2.5-\mathrm{kHz}$ comparison and $30 \mathrm{~Hz}$ for the $8-\mathrm{kHz}$ comparison frequencies. In the tactile task, these errors were much larger, representing about $3.0 \%$ of the $20-\mathrm{Hz}$ comparison frequency for the corrected procedure and over $8.0 \%$ for the uncorrected procedure. This is due primarily to the $\mathbf{S} 2$ frequency step size of $1 \mathrm{~Hz}$.
In summary, the safe-rate correction procedure does not significantly alter the measurement of threshold over the limited range of false-positive rates seen in the procedure described above. However, this procedure would result in large errors of threshold measurement with substantially larger false-positive rates (above 0.15 ). It may be possible to continue to use this procedure if the difference in frequency of the $\mathbf{S 2}$ stimuli is sufficiently small and if the psychometric function near the 0.50 level is sufficiently steep, although it would be imperative to verify the reliability of the method under these conditions. The uncorrected procedure is probably inadequate under most experimental conditions.

\section{DISCUSSION}

\section{Thresholds}

Tactile discrimination thresholds for 6 monkeys and auditory discrimination thresholds for 3 monkeys have been derived to date. The psychophysical performance and the estimated thresholds as a function of training in these monkeys are described in detail elsewhere (Recanzone, Jenkins, et al., 1991). The tactile discrimination thresholds estimated by this procedure were $2.34 \pm 0.47 \mathrm{~Hz}$ $(M \pm S D)$ with respect to a $20-\mathrm{Hz}$ comparison stimulus. One animal was also trained with a 30-Hz comparison stimulus. This trained animal had a threshold of $1.60 \pm 0.17 \mathrm{~Hz}$, taken over three consecutive sessions. The animals trained on the auditory discrimination task used either an $\mathbf{8 - \mathbf { k H z }}$ or a $2.5-\mathrm{kHz}$ stimulus as the comparison frequency. These animals had discrimination thresholds of $127.7 \mathrm{~Hz}$ for the $8-\mathrm{kHz}$ comparison frequency and $33.25 \mathrm{~Hz}$ for the $2.5-\mathrm{kHz}$ comparison frequency. One monkey was trained at both frequencies and had thresholds of 109.3 and $22.8 \mathrm{~Hz}$ for the $8-$ and $2.5-\mathrm{kHz}$ comparison frequencies, respectively.

The tactile thresholds measured as a percent of the comparison frequency ranged from $8.1 \%$ to $16.7 \%$, with a mean value of $11.6 \%$ for the tactile thresholds. The auditory thresholds were much better, ranging from $0.9 \%$ to $1.6 \%$, with a mean value of $1.2 \%$. 

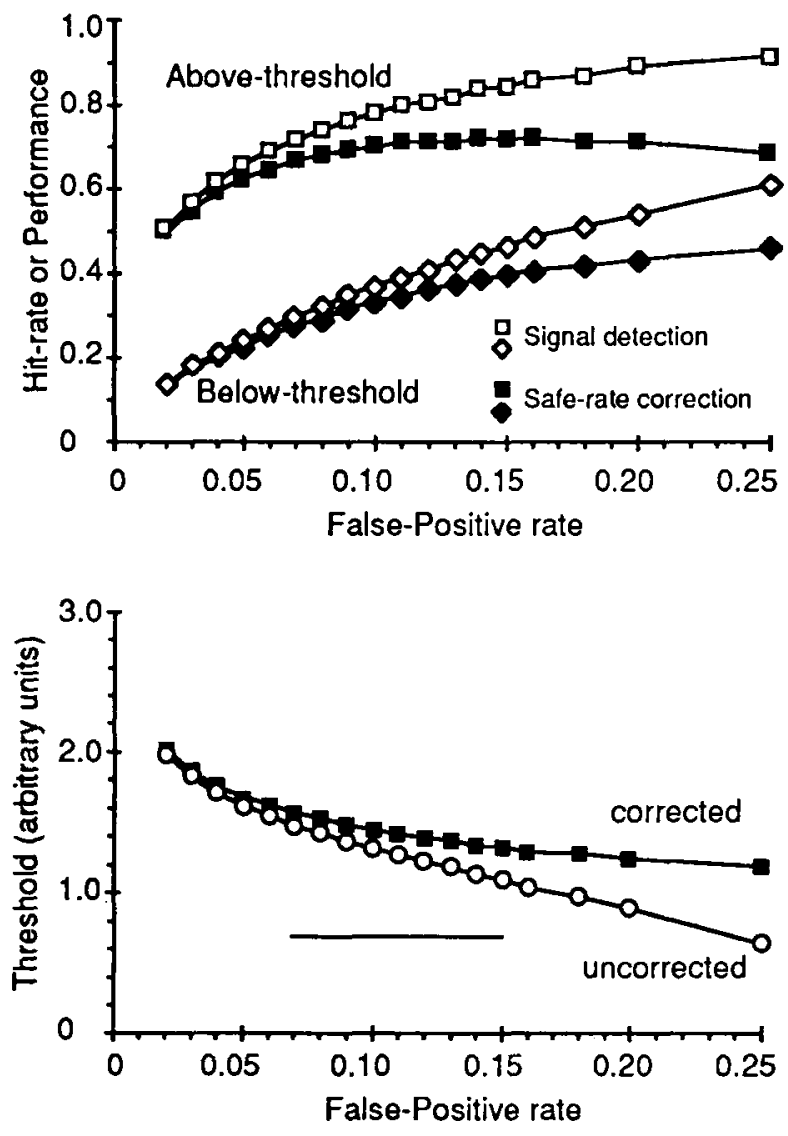

Figure 4. Comparison of the hit rates calculated with signaldetection procedures and the performance calculated with the saferate correction procedure. Top panel: The curve defined by the open squares represents the hit rate at each faise-positive rate that has the same $d^{\prime}$ as the standard above-threshold hit rate for representative case 1320; the open diamonds represent the same $d^{\prime}$ as the standard below-threshold hit rate for the same case. The filled symbols show the performance value obtained at the same hit rates at each false-positive rate. There is generally good agreement between these two measures at low false-positive rates. Bottom panel: Curves showing the corrected (squares) and uncorrected (diamonds) threshold measured for the performance and hit rates shown in the top panel. Threshold was taken in arbitrary units of step size, with the below-threshold performance or hit rate given a value of 1.0, and the above-threshold rate given a value of 2.0 . A perfect correction would generate a line with zero slope. The horizontal line denotes the range of false-pasitive rates seen in over $90 \%$ of the sessions for this example.

\section{Comparison to Other Paradigms}

This procedure has currently been used to define tactile frequency discrimination thresholds for up to 131 sessions in 6 adult owl monkeys, and auditory frequency discrimination thresholds for up to 118 sessions in 3 monkeys. We have been able to quantify the threshold estimated on daily sessions for each individual animal throughout the course of training and testing.

The tactile thresholds derived in this paradigm are in general agreement with those achieved in a two-aiternative forced-choice paradigm in macaques and humans (LaMotte \& Mountcastle, 1975; Mountcastle, Steinmetz, \& Romo,
1990; Mountcastle, Talbot, Sakata, \& Hyvarinen, 1969) at $20-$ and $30-\mathrm{Hz}$ comparisons, and they are similar to those derived in humans with the use of a $25-\mathrm{Hz}$ comparison (Goff, 1967). The auditory thresholds were slightly lower than those determined with a similar method in Old World monkeys, using a 2-kHz standard (Sinnott, Owren, \& Petersen, 1987) or a $1-\mathrm{kHz}$ standard (Sinnott, Petersen, $\&$ Hopp, 1985). They were also in agreement with those derived in human infants, but they were higher than those derived in human adults (Sinnott \& Aslin, 1985; Sinnott et al., 1985; Wier, Jesteadt, \& Green, 1977).

This procedure has advantages over a multiple-alternative forced choice in that the chance performance is low, yet the subject is not required to remember the sequence of stimulus frequencies or multiple response requirements. Animals performed 500-800 trials each session during the training and testing procedure, and they performed consistently, with over 100 trials in which the S2 frequency was below the estimated threshold. This allowed us to define thresholds in a difficult tactile task at which the subject could only cue on the temporal features-that is, the frequency-of the stimulus.

An alternative to the performance measure described in this report is to analyze the data by using the theory of signal detection (Green \& Swets, 1966) for each presented $S 2$ frequency. A measure of $d^{\prime}$ can be calculated for each $S 2$ frequency for each session.

\section{Possible Pitfalls}

We have encountered a number of strategies that result in fairly high reward rates even though the subject is not under strict stimulus control. The two most common strategies we term the guessing and the late-guessing strategies. These strategies could be effective for the subject because of the nature of the selection of the bin of presentation of the $\mathbf{S} 2$ frequency. If a false-positive response occurred in Bin 2, a time-out was presented, the lookup table was incremented, and the next run could then be initiated. Thus, if the subject always responded in Bin 2, the $S 2$ would eventually be presented, the response would be counted as a hit, and a reward would be delivered. If the false-positive time-out was sufficiently short, some animals were content at this schedule of reinforcement (0.37; see Table 1$)$. This strategy was quickly recognized by: (1) a high overall false-positive rate, (2) a relatively flat performance function, and (3) a high false-positive rate in Bin 2. This strategy was commonly adopted early in training and was prevented by: (1) increasing the falsepositive time-out, (2) presenting only $\mathbf{2} 2$ frequencies with large $\Delta f$, and (3) increasing the miss time-out. These parameter changes encouraged the subject to wait for the very obvious $\mathbf{S} 2$ stimulus in order to avoid lengthy time-outs.

In the late-guessing strategy, the subject would respond correctly to high $\Delta f \mathrm{~S} 2$ presentations in the early stimulus bins, but would also respond following a certain amount of elapsed time, usually in Bins 3 or 4, regardless of the stimulus presented. The performance using this more sophisticated guessing strategy was deceptively 
good, since most S2 presentations in Bin 3 were counted as hits, including those well below threshold. Because most stimulus presentations occurred in Bins 2 and 3 (see Table 1), the false-positive rate was also reasonably low. Two indicators were used to recognize this strategy: (1) The distribution of responses/bin was strongly skewed to the early bins; the animal usually released by Bin 4 , so that no stimuli were presented in Bins 5 and 6. (2) There was a high hit rate for catch trials (S2 $\Delta f=0$ ).

This strategy was discouraged by: (1) altering the probability of S2 occurrence in Bin 2 to $<10 \%$, (2) only presenting $\mathrm{S} 2$ frequencies in which the performance was $>90 \%$, (3) discontinuing the $S 2=S 1$ catch trials, and (4) increasing the false-positive time-out while decreasing the miss time-out.

An additional solution may be to not increment the lookup table following a false positive. In this way, the S2 would not eventually occur in Bin 2, but only in the originally designated bin. This strategy can be effective if care is taken to prevent the animal from making a long series of consecutive false-positive responses, which can occur if the $S 2$ is to be presented in Bin 5 or 6 . These animals would usually stop working under these conditions.

Our experience indicates that these animals adopted a specific strategy early in training and were very reluctant to alter this strategy once it was established. This was a desirable quality if the strategy was discrimination of the stimulus frequency, although that was not always the case. Thus, it was extremely important to maintain stimulus control over the animal in the early stages of training in order to facilitate the development of the desired discrimination behavior.

A second class of concerns was that the monkeys used intensity or subjective intensity cues and not the difference in frequency per se to perform the task. In the auditory paradigm, the sound field in the area occupied by the monkey's head was defined for the presented frequencies. This sound field was fairly uniform, and it had an overall range of intensities of approximately $12 \mathrm{~dB}$ SPL. In this free sound-field apparatus, the monkey could potentially move its head in a manner that would enhance intensity differences as a function of frequency. We believe this not to have been the case for several reasons. First, by presenting the two tone pips of a pair with only a 50msec separation, the ability of a subject to move its head within the sound field between pairs was limited. In addition, because the amplitude of each pip of the tone pip pair was randomly varied over an equivalent range as measured in the sound field, slight differences in intensity between pips virtually always occurred and thus could not be used as a reliable cue. Finally, videotaped and observed movements of the monkeys during performance of the task showed that the animals did not make head movements that were correlated with the stimulus presentations.

In the tactile task, stimuli were presented at the same amplitude at all frequencies. Previous work has shown that in the frequency range of $20-40 \mathrm{~Hz}$, there is a substantial difference in the "subjective intensity" of the stimuli (e.g., see von Békésy, 1959; see also Goff, 1967). LaMotte and Mountcastle (1975), who defined the subjective intensity curves for a $30-\mathrm{Hz}$ standard in both macaque monkeys and humans, found that at amplitudes of $200 \mu \mathrm{m}$, an intensity shift of approximately $20 \mu \mathrm{m}$ was necessary for a $4-\mathrm{Hz}$ difference in frequency. Unfortunately, no work has been done with the owl monkey to determine the behavioral thresholds of amplitude discriminations of sinusoidal stimuli at amplitudes in the range of $300 \mu \mathrm{m}$, as were used in this study. Subjective intensity differences with frequency are believed to result from an increase in the discharge rates of neurons as more cycles of the stimulus are presented during a given unit of time (see von Békésy, 1959). Because we were interested in potential neural correlates in the cerebral cortex to frequency discrimination, we chose not to correct for the potential cue of subjective intensity, reasoning that an available subjective cue should be reflected in the discharge of cortical neurons. In fact, the cycle-for-cycle firing of the cortical neurons accurately reflected the monkey subject's ability to discriminate frequency, whereas the discharge rate of the neurons did not reflect the behavioral performance (Recanzone, Merzenich, \& Schreiner, 1991; see also LaMotte \& Mountcastle, 1975; Mountcastle et al., 1990; Mountcastle et al., 1969; Talbot, DarianSmith, Kornhuber, \& Mountcastle, 1968). In order to control for subjective intensity, it would be necessary to define the equal subjective-intensity curves for the frequencies to be tested. This could be accomplished with the paradigm as described, simply by training the monkeys on a task at which they must respond to changes in the amplitude of the stimulus regardless of the frequency.

\section{Potential Uses}

The procedure described here can be used to derive psychophysical thresholds in a difficult sensory discrimination task. We were interested in examining the effects of attended versus unattended stimulation on the representation of these stimuli in the cerebral cortex. This was a major consideration in designing the temporal pattern of stimulus presentation. The $650-\mathrm{msec}$ duration of the tactile task was chosen because this duration is not long enough to result in adaptation of the firing rate of peripheral afferents or cortical neurons and it allows up to six stimulus presentations in a relatively short time, yet this duration is still long enough to provide extensive stimulation of the skin during the course of training. Similarly, the shorter but more frequent tone pips in the auditory task provide extensive stimulation at these frequencies, and they are an effective stimulus for activating auditory cortical neurons in this species (Imig et al., 1977). The timing of the stimulus presentations presented in this report could be easily modified to suit the specific issues being addressed. Indeed, this procedure could also be used 
to define the optimal timing of the stimulus presentations for sensory discrimination through alteration of the durations of the stimuli and the interstimulus intervals.

The procedure can also be simplified to define thresholds for other stimulus qualities. For example, one could easily set the $\mathbf{S 2}$ as a variation of amplitude, define Weber functions for different frequency-amplitude pairs, or vary the pause between stimulus intervals. In addition, eliminating the requirement of exact hand position simplifies the training procedure and would allow a more robust stimulus to be applied to a large portion of the hand. For example, vibration detection and discrimination thresholds could be defined by stimulation of the entire hand mold. When the procedure is used for other sensory modalities, the hand-placement contingencies can be relaxed to the more conventional leverpress or contact.

This procedure has an advantage over other paradigms in that multiple sensory modalities can be stimulated simultaneously while the subject only discriminates the stimulus of one modality. We have used this strategy to compare the effects of extended discrimination training with the effects of the same stimulus presentation regime in which no discriminations were made (passive stimulation) on changes in the functional organization of primary somatosensory cortex (Recanzone, Jenkins, et al., 1991; Recanzone, Merzenich, \& Jenkins, 1991; Recanzone, Merzenich, et al., 1991; Recanzone, Merzenich, \& Schreiner, 1991). As interest in the consequences of training and experience of sensory stimuli on the functional and anatomical organization of the central nervous system increases, this type of control procedure becomes critical for our understanding of these processes. In addition, one can dissect the performance as a function of training by assessing the thresholds of stimulated and trained skin surfaces from equivalent, unstimulated skin surfaces (see Recanzone, Jenkins, et al., 1991).

Psychophysical paradigms have been designed to test the acuity of the primate somatosensory system to discriminate spatial resolution (Johnson \& Phillips, 1981; Vierck \& Jones, 1970), absolute location (Vierck, Favorov, \& Whitsel, 1988), motion (Essick \& Whitsel, 1985a, 1985b), shape (Cole \& Glees, 1954; Johnson \& Phillips, 1981), texture (Carlson, 1981, 1984a; Lamb, 1983), and frequency (LaMotte \& Mountcastle, 1975; Mountcastle et al., 1990; Mountcastle et al., 1969). Studies in which attempts have been made to correlate the functional properties of the cerebral cortex with these psychophysically defined measures of acuity have been restricted to either ablation experiments (Burton, Sathian, \& Dian-Hua, 1990; Carlson, 1981, 1984b, 1984c; Carlson \& Burton, 1988; Cole \& Glees, 1954; LaMotte \& Mountcastle, 1979; Pons et al., 1988; Randolph \& Semmes, 1974; Semmes \& Porter, 1972; Semmes, Porter, \& Randolph, 1988), or measurements of the response properties of neurons of either welltrained animals performing the task (Essick \& Whitsel, 1985a, 1985b) or normal animals during equivalent stimulation paradigms (LaMotte \& Mountcastle, 1975; Vierck et al., 1988). These studies provide important information about the properties of responses of neurons within the cerebral cortex to these stimuli. The paradigm described above can also be used to investigate the effects of training a specific sensory surface on the neural responses at varying levels of the central nervous system by using either single-unit recording in the behaving animal (Mountcastle et al., 1990) or multiunit mapping techniques (Recanzone, Merzenich, \& Jenkins, 1991; Recanzone, Merzenich, et al., 1991; Recanzone, Merzenich, \& Schreiner, 1991).

Finally, adaptation of this procedure to humans only requires the substitution of an appropriate hand mold and reward. With human subjects, there is the advantage that training in the task can be done in the absence of stimulation (written instructions), and sensory modalities can be altered on command. With experimental animals, there is the advantage that invasive procedures can be used to define neurophysiological and/or anatomical correlates of the behavioral performance. Comparing results from both types of subjects, with the caveats of cross-species assumptions, may be a fruitful approach to the elucidation of the neural mechanisms of perception and improvements in performance with training.

\section{REFERENCES}

ALLMan, J. M., KAAs, J. H. (1975). The dorsomedial cortical visual area: $A$ third tier area in the occipital lobe of the owl monkey (Aotus trivirgatus). Brain Research, 100, 473-487.

Allman, J. M., \& KaAs, J. H. (1976). Representation of the visual field on the medial wall of occipital-parietal cortex in the owl monkey. Science, 191, 572-575.

ANDERSON, J. R. (1981). Cognitive skills and their acquisition. Hillsdale, NJ: Erlbaum.

Burton, H., Sathian, K., \& Dian-Hua, S. (1990). Altered responses to cutaneous stimuli in the second somatosensory cortex following lesions of the postcentral gyrus in infant and juvenile macaques. Joumal of Comparative Neurology, 291, 395-414.

CARLSON, M. (1981). Characteristics of sensory deficits following lesions of Brodmann's areas 1 and 2 in the postcentral gyrus of Macaca mulatta. Brain Research, 204, 424-430.

CARLSON, M. (1984a). Development of tactile discrimination capacity in Macaca mulatta: I. Normal infants. Developmental Brain Research, 16, 69-82.

Carlson, M. (1984b). Development of tactile discrimination capacity in Macaca mulatta: II. Effects of partial removal of primary somatic sensory cortex $(\mathrm{Sml})$ in infants and juveniles. Developmental Brain Research, 16, 83-101.

Carlson, M. (1984c). Development of tactile discrimination capacity in Macaca mulatta: III. Effects of total removal of primary somatic sensory cortex (Sml) in infants and juveniles. Developmental Brain Research, 16, 103-117.

Carlson, M., Burton, H. (1988). Recovery of tactile function after damage to primary or secondary somatic sensory cortex in infant Macaca mulatta. Journal of Neuroscience, 8, 833-859.

Chubbuck, J. G. (1966, May-June). Small-motion biological stimulator. Applied Physics \& Laboratory Techniques Digest, pp. 18-23.

COLE, J., \& GLES, P. (1954). Effects of small lesions in sensory cortex in trained monkeys. Journal of Neurophysiology, 1, 1-13.

Essick, G. K., \& WHTSEL, B. L. (1985a). Assessment of the capacity of human subjects and SI neurons to distinguish opposing directions of stimulus motion across the skin. Brain Research Reviews, 10, 187-212.

Essick, G. K., \& WhitseL, B. L. (1985b). Factors influencing cutaneous directional sensitivity: A correlative psychophysical and neurophysiological investigation. Brain Research Reviews, 10, 213-230. 
GiBson, E. J. (1953). Improvement in perceptual judgments as a function of controlled practice or training. Psychological Bulletin, 50, $401-431$.

GoFf, G. D. (1967). Differential discrimination of frequency of cutaneous mechanical vibration. Joumal of Experimental Psychology, 74, 294-299.

GREEN, D. M., \& SWETS, J. A. (1966). Signal detection theory. New York: Wiley.

ImgG, T. J., Ruggero, M. A., Krtzes, L. M., JAvel, E., \& Brugge, J. F. (1977). Organization of auditory contex in the owl monkey (Aotus trivirgatus). Journal of Comparative Neurology, 171, 111-128.

JAMES, W. (1950). The principles of psychology (Vol. 1). New York: Dover. (Original work published 1890)

JENKINS, W. M. (1982). Interfacing the Apple II for the behavioral laboratory. Behavior Research Methods \& Instrumentation, 14, 345-347.

Jenkins, W. M., Merzenich, M. M., OChs, M., Allard, T. T., GUICE-Rosles, E. (1990). Functional reorganization of primary somatosensory cortex in adult owl monkeys after behaviorally controlled tactile stimulation. Journal of Neurophysiology, 63, 82-104.

Johnson, K. O., \& Pillips, J. R. (1981). Tactile spatial resolutions: I. Two-point discrimination, gap detection, grating resolution and letter recognition. Journal of Neurophysiology, 46, 1177-1191.

KaAs, J. H., Krubitzer, L. H., Chino, Y. M., Langston, A. L., Polley, E. H., Blair, N. (1990). Reorganization of retinotopic cortical maps in adult mammals after lesions of the retina. Science, 228, 229-231.

KaAs, J. H., Nelson, R. J., Sur, M., Chia-Sheng, L., \& Merzenich, M. M. (1979). Multiple representations of the body within the primary somatosensory cortex of primates. Science, 204, 521-523.

LAMB, G. D. (1983). Tactile discrimination of textured surfaces: Psychophysical performance measurements in humans. Journal of Physiology, 338, 551-565.

LAMOTTE, R. H., \& Mountcastle, V. B. (1975). Capacities of humans and monkeys to discriminate between vibratory stimuli of different frequency and amplitude: A correlation between neural events and psychophysical measurements. Joumal of Neurophysiology, 38, 539-559.

LaMotte, R. H., \&ountcastle, V. B. (1979). Disorders in somesthesis following lesions of parietal lobe. Journal of Neurophysiology, 42, 400-419.

Merzenich, M. M., KAAS, J. H. (1980). Principles of organization of sensory-perceptual systems in mammals. Progress in Psychobiology \& Physiological Psychology, 9, 1-42.

Merzenich, M. M., KAAs, J. H., SUR, M., \& Ln, C. S. (1978). Double representation of the body surface within cytoarchitectonic areas $3 b$ and 1 in "SI" in the owl monkey (Aotus trivirgatus). Joumal of Comparative Neurology, 181, 41-73.

Merzenich, M. M., Nelson, R. J., KaAs, J. H., Stryker, M. P., Jenkins, W. M., Zook, J. M., Cynader, M. S., Schoppman, A. (1987). Variability in hand surface representations in areas $3 \mathrm{~b}$ and 1 in adult owl and squirrel monkeys. Joumal of Comparative Neurology, 258, 281-296.

Merzenich, M. M., Recanzone, G. H., Jenkins, W. M., Allard, T. T., NuDO, R. J. (1988). Cortical representational plasticity. In T. P. Rakic \& W. Singer (Eds.), Neurology of neocortex (pp. 41-67). New York: Wiley.

Merzenich, M. M., Recanzone, G. H., JenKINS, W. M., \& Nudo, R. J. (1990). How the brain functionally rewires itself. In M. Arbib \& J. A. Robinson (Eds.), Natural and artificial parallel computations (pp. 177-210). Cambridge, MA: MIT Press.

Mountcastle, V. B., Steinmetz, M. A., \& Romo, R. (1990). Frequency discrimination in the sense of flutter: Psychophysical measurements correlated with postcentral events in behaving monkeys. Journal of Neuroscience, 10, 3032-3044.

Mountcastle, V. B., Talbot, W. H., Sakata, H., Hyvarnen, J. (1969). Cortical neuronal mechanisms in flutter-vibration studied in unanesthetized monkeys. Neuronal periodicity and frequency discrimination. Journal of Neurophysiology, 32, 452-484.
Pons, T. P., Garraghty, P. E., Mishkin, M. (1988). Lesion-induced plasticity in the second somatosensory cortex of adult macaques. Proceedings of the National Academy of Science, 85, 5279-5281.

RANDOLPH, M., \& SEmmes, J. (1974). Behavioral consequences of selective subtotal ablations in the postcentral gyrus of Macaca mulatta. Brain Research, 70, 55-70.

Recanzone, G. H., Jenkins, W. M., Hradek, G. T., Merzenich, M. M. (1991). Progressive improvements in discriminative abilities in adult owl monkeys performing a tactile frequency discrimination task. Manuscript submitted for publication.

Recanzone, G. H., Merzenich, M. M., Jenkins, W. M. (1991). Frequency discrimination training engaging a restricted skin surface results in an emergence of a cutaneous response zone in cortical area $3 a$. Manuscript submitted for publication.

Recanzone, G. H., Merzenich, M. M., Jenkins, W. M., Grajsi, K. A., DINSE, H. R. (1991). Topographic reorganization of the hand representation in cortical area $3 b$ of owl monkeys trained in a frequency discrimination task. Manuscript submitted for publication.

Recanzone, G. H., Merzenich, M. M., Schreiner, C. E. (1991). Changes in the distributed temporal response properties of SI cortical neurons reflect improvements in performance on a temporally based tactile discrimination task. Manuscript submitted for publication.

Robertson, D., IRVINE, D. R. F. (1989). Plasticity of frequency organization in auditory cortex of guinea pigs with partial unilateral deafness. Joumal of Comparative Neurology, 282, 456-471.

SAnes, J. N., Suner, S., Donoghue, J. P. (1990). Dynamic organization of primary motor cortex output to target muscles in adult rats: I. Long-term patterns of reorganization following motor or mixed peripheral nerve lesions. Experimental Brain Research, 79, 479-491.

SEMmes, J., PORTER, L. (1972). A comparison of precentral and postcentral cortical lesions on somatosensory discrimination in the monkey. Cortex, 8, 249-264.

Semmes, J., Porter, L., RANdolph, M. (1974). Further studies of anterior postcentral lesions in monkeys. Cortex, 10, 55-68.

SingleY, M. K., \& ANDERSON, J. R. (1989). The transfer of cognitive skill. Cambridge, MA: Harvard University Press.

SinNotT, J. M., \& AsLin, R. N. (1985). Frequency and intensity discrimination in human infants and adults. Joumal of the Acoustical Society of America, 78, 1986-1992.

Sinnott, J. M., Owren, M. J., Petersen, M. R. (1987). Auditory frequency discrimination in primates: Species differences (Cercopithecus, Macaca, Homo). Joumal of Comparative Psychology, 101, 126-131

Sinnott, J. M., Petersen, M. R., Hopp, S. L. (1985). Frequency and intensity discrimination in humans and monkeys. Joumal of the Acoustical Sociery of America, 78, 1977-1985.

Talbot, W. M., Darian-Smith, I., Kornhuber, H. H., MountCASTLE, V. B. (1968). The sense of flutter-vibration: Comparison of the human capacity with response patterns of mechanoreceptive afferents from the monkey hand. Joumal of Neurophysiology, 31, 301-334.

VIERCK, C. J., JR., FAvorov, O., \& WhITSEL, B. L. (1988). Neural mechanisms of absolute tactile localization in monkeys. Somatosensory \& Motor Research, 6, 41-61.

VIERCK, C. J., JR., JoNes, M. B. (1970). Influences of low and high frequency oscillation upon spatio-tactile resolution. Physiology \& Behavior, 5, 1431-1435.

voN BÉKÉsY, G. (1959). Synchronism of neural discharges and their demultiplication in pitch perception on the skin and in hearing. Journal of the Acoustical Society of America, 31, 338-349.

Wier, C. C., Jesteadt, W., Green, D. M. (1977). Frequency discrimination as a function of frequency and sensation level. Joumal of the Acoustical Society of America, 61, 178-184.

(Manuscript received November 20, 1990; revision accepted for publication March 25, 1991.) 